\title{
LOS ESTUDIANTES DE CICLO BÁSICO Y LAS ESTRATEGIAS DE APRENDIZAJE
}

Mirta Antelo*
Virginia Hill
Dora Laranjo

\section{Propósito}

En las más diversas ocasiones, tales como salas docentes, coordinaciones, reuniones de profesores, encuentros ocasionales, es común escuchar las expresiones: "los alumnos no saben estudiar", "los estudiantes no comprenden lo que leen", "solos no consiguen razonar y resolver los problemas que se les presentan". Todo esto, sumado a nuestra propia vivencia como docentes, nos ha llevado a pensar que en general, se perciben dificultades y frustraciones en el proceso de aprendizaje de los alumnos del Ciclo Básico de Enseñanza Secundaria, agudizándose éstas al cursar el Bachillerato (tanto Bachillerato Diversificado como Bachillerato Tecnológico).

Considerando las expresiones antes mencionadas de los docentes, nos proponemos identificar las formas de aprender que ponen en juego los alumnos que están terminando el 3er. año del Ciclo Básico (entre 14 y 16 años), al ser enfrentados a situaciones de la vida cotidiana o de aprendizaje. Entendemos relevante abordar este tema, porque podría aportar a los profesores elementos para replantearse la forma de enseñar, procurando así potenciar y desarrollar en los alumnos mejores formas de aprender. Preocupados por la situación de los estudiantes, con respecto a su aprendizaje, es que nos planteamos la siguiente pregunta: ¿Qué estrategias de aprendizaje han logrado los alumnos que están cursando el 3er. año de Ciclo Básico?

\section{Marco conceptual}

Llevar adelante la investigación sobre las estrategias de aprendizaje logradas por los alumnos del Ciclo Básico, exige precisar una serie de conceptos que ilustren desde qué óptica o concepción teórica se la enfocará y, por ende, cuál será el marco teórico que la sustentará. Resulta necesario tener presente que el concepto de ciencia ha ido variando a través del tiempo. Así mismo, el saber científico, no sólo influye sino que también es influido por la realidad sociocultural y el momento histórico en el que se inserta. Es por ello que la ciencia hoy, en un momento en que la teoría social contemporánea se encuentra en crisis porque las perspectivas teóricas de la "postmodernidad" la han movilizado, desestructurado, debe enfrentarse a la "supremacía crítica" y la "desconstrucción" que hacen tambalear a las epistemologías basadas en los principios fundamentales. A su vez, el rechazo de los fundamentos básicos de la racionalidad, condujo al destronamiento de la autoridad de la ciencia positivista. Carentes así, de un marco normativo que indique qué deben construir y cómo deben orientar su práctica científica, las distintas ciencias han estado viviendo una especie de "blanco intelectual", que Habermas (1985) llama "descontruccionismo de cabriolas". Obviamente, la enseñanza de las ciencias no puede evadir esta situación en la que el saber científico ya no es más visto como verdad absoluta, incuestionable; el relativismo, el cambio permanente, la incertidumbre, también han invadido el ámbito educativo. 
Y es precisamente ante esta realidad sociocultural y científica, que la enseñanza debe tomar postura y optar por una concepción del aprendizaje que posibilite al estudiante la comprensión de esta "nueva" ciencia, además de su adecuada inserción en esta sociedad en la que reinan el cambio, la incertidumbre y la desconstrucción. Por tal razón efectuaremos la investigación a la luz de una concepción constructivista de la enseñanza de la ciencia, que no considera a los alumnos "tabulas rasas", sino seres poseedores de múltiples concepciones, representaciones que forjadas a la luz de sus vivencias, del conflicto entre el lenguaje cotidiano y el científico, del refuerzo de la cultura, conforman una trama conceptual personal, de gran arraigo y de fuerte coherencia interna, para explicar los fenómenos naturales.

Así concebidos los procesos de enseñanza y de aprendizaje, resulta inminente plantear situaciones significativas para el educando, que lo lleven a confrontar sus constructos con la nueva información procurando generar un conflicto cognitivo que pueda inducir a un cambio conceptual. Para ello resulta de vital importancia dar un enfoque holístico a los temas, generar espacios de reflexión individual y colectiva, promover la realización de tareas de campo, experimentos, investigaciones, que permitan al estudiante aplicar sus saberes, potenciar sus capacidades, reforzar su autoestima. Es en esta permanente dialéctica que podrá tomar conciencia de la realidad, de los problemas del medio en que vive y actuar como ciudadano libre y responsable. Esta visión del proceso nos permite actualizar, acomodar a la realidad reinante, redefinir, redimensionar el concepto de aprendizaje, concepto que ya no puede entenderse como mera repetición o respuesta a la información recibida sino que, por el contrario, debería orientarse hacia objetivos y considerar el "deseo" y la "motivación" como motores del proceso. Así mismo, en un mundo en el que la información llega por tantas vías, el aprendizaje tal vez debe hacer hincapié en seleccionar, jerarquizar, organizar la información y redimensionar la memoria, no como acopio de datos sino como integración de información para la elaboración de futuros proyectos. Sin duda, desde esta concepción del aprendizaje de las ciencias, es necesario revalorizar el logro y empleo de variadas estrategias cognitivas y metacognitivas. Para reafirmar nuestra postura nos parece interesante citar la siguiente definición: "Es relativamente nuevo en la teoría de la educación, conceptualizar el aprendizaje como pensamiento, es decir, usando los conocimientos previos y las estrategias específicas para entender las ideas de un texto como totalidad o los elementos de un problema como totalidad" (JONES y otros. 1987: 21). Definido el aprendizaje como "pensamiento", debemos admitir que si bien se da en etapas, no es lineal sino recursivo, lo que implica que muchas veces debemos recordar lo dado para poder asociar, comparar, relacionar y así avanzar. Ya Vigotsky sostenía que cuando conectamos una idea nueva con algo familiar, podemos tener que indagar en la memoria y verificarla, o vincularla con otra cosa y así repensarla (Vigotsky, 1992)

En la medida en que, pueda lograrse un proceso de aprendizaje con las características antes mencionadas, correspondería que el educando tomara conciencia acerca de qué aprende y cómo aprende. Ante esta interrogante surgen los conceptos de estrategias de aprendizaje y de metacognición que no pueden introducirse en forma aislada uno de otro, sino que hacen referencia mutuamente.

Precisamente, el concepto de metacognición es uno de los aportes más importantes de la psicología cognitiva. Se refiere a la toma de conciencia y al control que los individuos tienen sobre sus procesos cognitivos. La regulación metacognitiva requiere el uso de estrategias tales como la planificación de acciones, la verificación de los resultados, la superación de dificultades, que permitan controlar la tarea y los esfuerzos cognitivos. Estas estrategias generales pueden ser aplicadas, no sólo en la resolución de problemas específicos, sino en diferentes situaciones de la vida cotidiana, por lo que, además de favorecer el aprendizaje, ayuda al estudiante a "calibrar" el éxito de sus esfuerzos. La metacognición estimula también la confrontación de los conocimientos previos con la nueva información. Tal como expresa Meirieu, la metacognición es la "... actividad mediante la cual el sujeto se cuestiona acerca de las estrategias de aprendizaje y relaciona los medios utilizados con los resultados obtenidos; puede de este modo estabilizar unos procedimientos dentro de unos procesos..." (Meirieu, 1992: 207). 
A su vez, si consideramos que las habilidades metacognitivas son las que permitirán utilizar y controlar el conocimiento de otras habilidades cognitivas ayudando así a planificar y regular con eficacia el empleo de los propios recursos cognitivos, resulta evidente que las estrategias metacognitivas implicarían un aprendizaje de tipo superior, un metaprendizaje, ya que pueden aplicarse en cualquier situación y disciplina de estudio. Visto así, un alumno que haya desarrollado estrategias metacognitivas sería capaz de relacionar la nueva información con lo ya aprendido; podría conocer, juzgar y controlar su proceso de comprensión al leer, al escuchar, al memorizar, al resolver problemas e incluso explicitar el uso de las estrategias. En definitiva, sería consciente de cómo aprende y en qué circunstancias podría desplegar las estrategias.

Por su parte, Gibaja (1995) agrega al concepto de metacognición otro aspecto de real importancia para nuestro estudio - que toma como protagonistas a adolescentes del 3er. año del ciclo Básico- ya que afirma que después de muchos años de investigación, tiene la convicción de que el conocer acerca del conocer aumenta con la edad y la experiencia. Esto nos estaría indicando entonces, que los más jóvenes tienen un conocimiento más limitado de sus saberes que los mayores. Así mismo, sostiene que la adquisición de las estrategias de aprendizaje puede ser espontánea o adquirida mediante una enseñanza específica.

Entonces, para que pueda darse este proceso de metacognición, los estudiantes, deberán tomar conciencia de cuáles son las estrategias que ponen en juego en las diferentes situaciones de su proceso de aprendizaje, entendiendo por estrategias de aprendizaje el "modo de representación de la actividad cognitiva de los sujetos a partir de la descripción de los comportamientos intelectuales eficientes dentro de unas situaciones didácticas precisas. La estrategia de un sujeto se articula de este modo en un estilo cognitivo personal relativamente estable pero que depende también del objeto de aprendizaje" (Meirieu, 1992: 204).

Por su parte, Mayor y otros (1995), sostienen que "Estrategia es el conjunto de procedimientos que se instrumentan y se llevan a cabo para lograr algún objetivo, plan, fin o meta. Aplicado al aprendizaje es la secuencia de procedimientos que se aplican para lograr aprender" (Mayor y otros 1995: 29).

Si bien cada disciplina requiere del desarrollo de estrategias de aprendizaje específicas, existen también algunas que son comunes. Estas son las que refieren al proceso por el cual el estudiante obtiene, hace acopio y utiliza la información. Según las distintas situaciones, el estudiante pondrá en juego estrategias de: repaso simple, selección e interpretación de la información, búsqueda de analogías, análisis de la información y realización de inferencias, comprensión y organización de la información, comunicación de lo aprendido y control de la comprensión. Sobre la base de éstas, elaboraremos nuestras situaciones problema que permitirán la realización del relevamiento de las estrategias de aprendizaje logradas por los estudiantes. Las situaciones deberían constituir una forma de desafiarlos para encontrar respuestas, solución a fenómenos complejos, curiosos, inciertos, generando asimismo un profundo compromiso en la búsqueda del conocimiento. Bransford y Stein (1984) describen el llamado método IDEAL de Brandsford, que representa un modelo viable en la resolución de diferentes tipos de problemas. Precisamente cada una de las letras de la sigla alude a un paso de la resolución del problema. Así, la I se refiere a la Identificación; la D a la Definición y representación del problema; la $\mathbf{E}$ se alude a la Exploración de alternativas para la resolución; por último se llegaría a la Acción mediante la planificación y el logro de las potenciales soluciones que permitirían extraer conclusiones Lógicas acerca de lo actuado. Asimismo, en este proceso, el alumno debería hacerse preguntas y confrontar sus concepciones con la nueva información, para así lograr un cambio conceptual. Tal como lo establece Eduardo Martí la mayoría de los autores comparten la idea de que los alumnos deberían desarrollar procedimientos de resolución de problemas guiados de forma planificada y consciente, pasando así, "... de un conocimiento técnico y automático a un conocimiento estratégico" (en Pozo y Monereo, 1999: 113). 
Ahora bien, aunque nuestro trabajo pretende concentrarse en cómo aprenden los alumnos, no podemos dejar de lado las concepciones de enseñanza, las estrategias de enseñanza y las relaciones que pueden tener con los procesos de aprendizaje, ya que consideramos que es a través de las estrategias de enseñanza que se da el logro de estrategias de aprendizaje. Pero, si bien esto no será trabajado, nos parece relevante establecer el concepto de enseñanza, que guiará y encuadrará nuestra investigación. Concebimos la enseñanza como un proceso permanente, eminentemente humano, intencional, que procurará comunicar, informar, guiar, orientar, facilitar procesos, potenciar actitudes saludables y capacidades intelectuales, morales, espirituales; en un marco ético de participación real, plural, como práctica social que es y que trasciende las intenciones e intereses individuales para atender las exigencias, los requerimientos sociales del momento histórico en el que se inserta. Al decir de Contreras: "Si, como hemos visto, la enseñanza es una actividad intencional que obliga moralmente a sus responsables, también es una práctica social que excede a su comprensión como producto de decisiones individuales, generando una dinámica que sólo puede comprenderse en el marco del funcionamiento general de la estructura social de la que forma parte". (1999:17).

Lo expuesto por Contreras, reafirma la idea de que el alumno construye sí, sus conocimientos, pero no en soledad, sino en la interacción con sus pares, con la guía del docente, con el aporte de técnicas, de actividades, de estrategias de enseñanza que provoquen el logro de estrategias de aprendizaje, entendidas como "conjunto de operaciones realizadas por un sujeto con objeto de conseguir un aprendizaje estabilizado". (Meirieu, 1992: 149-150)

La curiosidad que también actúa como motor en el proceso de aprendizaje y por ende en la adquisición de las estrategias pertinentes, se ve debilitada al recibir desde temprana edad tanta información que excede incluso los requerimientos de la edad. Tal como sostiene Tedesco "El pasado es concebido como un obstáculo, lo cual nos coloca ante un escenario en donde el manejo de los aparatos por parte de los niños y no por los adultos crea la separación entre pensamiento y conocimiento. Los niños conocen y operan, pero no pueden pensar en el sentido de lo que hacen. Los adultos, en cambio, pueden pensar en el sentido pero no saben operar con los nuevos instrumentos". (1999: 49-50). Vemos entonces que la identidad de nuestros alumnos se construye en medio del bombardeo de los multimedias, sin por ello estar más acompañados en su crecimiento. Por el contrario, en lugar de retrotraerse y reflexionar sobre sí mismos, corren tras los estereotipos de moda, que también persiguen los adultos en un fenómeno de "adolescentización" que toma la adolescencia como un ideal a alcanzar y mantener, y la transforma así, en una etapa sin límites ni perfil propio.

No estamos negando en el adolescente actual la existencia de características propias de la etapa, ni tampoco su pasaje por los tan mentados "duelos" de este período de la vida, pero sin duda enfrenta otros más, acordes con las peculiaridades de la época en que viven. Por todo lo expuesto y analizado, no restamos importancia al razonamiento formal que en el caso de Piaget por ejemplo, establece que el desarrollo intelectual culmina en la adolescencia, cuando se alcanza el nivel de las operaciones formales, pero si deseamos comprender a nuestros educandos y la forma como logran sus estrategias de aprendizaje, no podemos desconocer el razonamiento informal que se aplica fuera de los contextos formales de las matemáticas y la lógica simbólica, cobrando real importancia el contexto y la situación en que se opera. Entendiendo entonces que el razonamiento informal se aplica en la vida cotidiana, apelando a actividades intelectuales tales como la planificación, la argumentación, el optar y jerarquizar conceptos, el establecer relaciones, causas y consecuencias, los pro y contra de ciertas situaciones, influidas por las creencias y teorías personales, así como por el conocimiento específico que le conferirá orden y claridad, no podemos desconocerlo a la hora de analizar las estrategias desplegadas por los alumnos investigados. 
Hoy por hoy, resulta indiscutible que en la resolución de problemas, de situaciones de la vida cotidiana y en el desempeño final del estudiante se entrelazan el razonamiento formal y el informal, conformando un conjunto de heurísticos, de "atajos" mentales más o menos eficaces, acorde con la posesión del conocimiento específico que le confiere al razonamiento una estructura más clara y ordenada. Es por ello, que para el estudio de las estrategias de aprendizaje de los alumnos del $3^{\text {er }}$ año del Ciclo Básico y el posterior análisis de los datos, deberíamos tener en cuenta, la multiplicidad de aspectos que interactúan constituyendo un complejo entramado en los procesos de enseñanza y de aprendizaje.

\section{Metodología}

Para indagar las estrategias de aprendizaje de los alumnos del Ciclo Básico, de acuerdo con lo expresado en el propósito, efectuamos un estudio de caso en el cual nos planteamos la siguiente pregunta de investigación:

Básico?

¿Qué estrategias de aprendizaje han logrado los alumnos que están cursando el $3^{\text {er }}$ año de Ciclo

Desde nuestra perspectiva, no pretendemos cuantificar ni generalizar, sino evaluar los procesos mediante los cuales los alumnos se apropian del conocimiento. A tales efectos, centramos nuestro trabajo en los estudiantes de $3^{\text {er }}$ Año de Ciclo Básico, obteniendo la información directamente de ellos a través de diferentes técnicas:

1. Resolución de situaciones problema.

2. Entrevistas.

Nos planteamos la resolución de situaciones problema, como forma de obtener la información, por considerar que al enfrentarlas, el alumno debería poner en juego las diversas estrategias antes mencionadas en el marco teórico (comprender, seleccionar, organizar...), que de otra forma no se pondrían de manifiesto con la misma claridad. A través de las entrevistas nos propusimos obtener del alumno, la explicitación de los procedimientos seguidos en la resolución de problemas, así como también, informarnos de las dificultades que encontró al resolverlos en soledad y que tal vez podría haberlas superado en la interacción con otros.

Para realizar el trabajo de investigación, seleccionamos intencionalmente alumnos pertenecientes a un instituto privado de enseñanza media, situado en la zona del Cordón, con una población identificada por el informe de MESyFOD como perteneciente al nivel socioeconómico medio - medio alto. Un 50\% de la población vive en el barrio y el otro $50 \%$ se distribuye en el resto del departamento, en su mayoría en zonas urbanas. La elección de este liceo fue pautada entre otros factores por: a) la accesibilidad al centro de estudio y b) ninguna de las participantes en la investigación ejerce docencia directa en el mismo.

Por lo expuesto en el párrafo anterior, al seleccionar todos los alumnos de un mismo instituto eliminamos las variables "nivel socioeconómico" y "tipo de centro" que se habrían presentado en el caso de trabajar con alumnos de diferentes centros. Tenemos presente que la forma de trabajo en un centro docente podría modificar el proceso de adquisición de estrategias de aprendizaje, pero dado que pretende ser un estudio de caso sin posibilidad de cuantificar ni comparar, se optó por esta modalidad.

Para elaborar las situaciones problema mencionadas anteriormente, tuvimos en cuenta los siguientes criterios: 
- Como eje temático: contenidos de Biología trabajados durante los cursos, así como temas relacionados con conocimientos generales y de la vida diaria.

- Se buscó que el diseño fuera atractivo y actual, que les planteara situaciones reales y cotidianas (a las que se pueden enfrentar al leer un periódico o escuchar un noticiero), o imaginarias pero con sentido científico.

- Los ejercicios propuestos serían resueltos por escrito y en forma individual.

Con relación a lo anterior, se elaboraron ocho situaciones problema que fueron testeados con estudiantes, pertenecientes a una institución privada de similares características, con la finalidad de comprobar su eficacia, verificar si los datos recabados eran relevantes para la investigación, si los alumnos los comprendían y así poder realizar los ajustes necesarios para la propuesta final. Se analizaron las respuestas dadas en primera instancia y de esto resultó la elección de cuatro situaciones problema.

Una vez terminada la etapa de testeo y ajustes, se solicitó a 40 alumnos que voluntariamente quisieran participar, la resolución de las cuatro situaciones problema. Al pedirles su colaboración en la investigación, se les explicó en un encabezado de la hoja, el objetivo perseguido por la misma. Para esta selección tuvimos en cuenta el nivel de corrección de las respuestas y el desempeño escolar de cada uno de los educandos -según información obtenida en la institución-, procurando una variada gama en el rendimiento de los mismos. Después de haber leído todos lo trabajos, considerando la saturación de las variables y el escaso tiempo con que contábamos, decidimos acotar nuestro estudio, seleccionando sólo seis alumnos, que serían los entrevistados.

A los seis alumnos seleccionados se los entrevistó con una pauta semiestructurada, procurando indagar los procesos y estrategias empleados en la resolución de las situaciones problema, intentando además, identificar las dificultades encontradas en cada etapa.

La finalización de los cursos, no nos permitió realizar observaciones de clase, como habíamos planteado en nuestro Proyecto. Aquellas tenían por finalidad ver en acción las estrategias de los alumnos en interacción con sus pares y con el docente, de una manera más natural, para así tener más elementos del proceso mediante el cual los estudiantes ponen en práctica las estrategias de aprendizaje.

\section{Análisis y discusión de datos}

Una vez culminada la recolección de datos, efectuada a través de las técnicas propuestas (resolución de situaciones problema y entrevistas), iniciamos el proceso de análisis de los mismos. Cabe destacar que no existe una única interpretación de los datos obtenidos, por lo que, la que presentamos es tan sólo una, que responde a los objetivos propuestos, al marco conceptual del que partimos, así como también a nuestra biografía escolar, nuestra formación profesional, nuestra socialización profesional, nuestra práctica docente, nuestra configuración didáctica y nuestra postura ante los procesos de enseñanza y de aprendizaje.

En un comienzo, para el análisis de los datos, nos inspiramos en los autores Taylor y Bogdan (1987). Así, leímos varias veces los trabajos, efectuamos múltiples observaciones, puntualizaciones para relevar aspectos y temas emergentes. Así mismo, procuramos: confeccionar tipologías, formular interpretaciones, extractar ideas, desarrollar conceptos y proposiciones teóricas acordes con nuestro marco conceptual, para extraer conclusiones válidas. Sin embargo, llegado a este punto de ardua labor, sentimos que la rica variedad de la información obtenida, nos desbordaba a la hora de intentar diseñar un instrumento adecuado, por lo que, sin perder flexibilidad, pensamos que debíamos auxiliarnos con algunos de los modelos de análisis de estrategias ya existentes. 
Al consultar diferentes autores, encontramos varias clasificaciones de estrategias de aprendizaje. Ante tal diversidad, comprendimos que afiliarnos a una categorización, podría limitar o forzar el análisis. Inspiradas en la clasificación que presenta Avolio de Cols en su libro "Los proyectos para el trabajo en el aula" (1998) que las agrupa según el propósito y la función que cada una de las estrategias cumple dentro del proceso de aprendizaje, y considerando que las estrategias son, según Mayor, "...conjunto de procedimientos que se instrumentan y llevan a cabo para lograr algún objetivo, plan, fin o meta" (1995: 29), elaboramos diversos instrumentos de análisis que nos permitieran identificar esos procedimientos. De ese modo, fuimos testeando diferentes matrices de análisis de datos hasta alcanzar el diseño final. En el mismo incluimos cinco grupos de estrategias, las cuales a su vez se subdividieron.

Una vez elaborada la matriz de datos fuimos tratando de identificar en las situaciones problema $\mathrm{y}$ en las entrevistas de los seis alumnos elegidos, las estrategias de aprendizaje que se presentan en el siguiente cuadro:

\section{Síntesis de las estrategias identificadas}

\begin{tabular}{|c|}
\hline 1. Interpretación de la información \\
\hline 1.1. Seleccionar la que es de utilidad \\
\hline 1.2. Codificar la información en un nuevo código \\
\hline 1.2.a. Técnico -cotidiano \\
\hline 1.2.b. Escrito-gráfico \\
\hline 1.2.c. Escrito-matemático \\
\hline 1.3. Manejarse dentro de un mismo código \\
\hline 1.4. Relacionar la nueva información con las ideas previas \\
\hline 2. Comprensión y organización conceptual de la información \\
\hline 2.1. Técnicas facilitadoras de la comprensión del discurso escrito \\
\hline 2.2. Relación de conceptos \\
\hline 2.3. Jerarquización de conceptos \\
\hline 3. Búsqueda de analogías para interpretar los fenómenos \\
\hline 4. Comunicación de la información \\
\hline 4.1. Expresión de la información en forma escrita \\
\hline 4.1.a. Respetar reglas de ortografía y sintaxis \\
\hline 4.1.b. Efectuar una introducción al tema \\
\hline 4.1.c. Asegurar la lógica del discurso \\
\hline 4.1.d. Producir argumentos coherentes \\
\hline 4.1.e. Citar ejemplos adecuados \\
\hline 4.2. Expresión de la información en forma oral \\
\hline 4.2.a. Respetar reglas de sintaxis \\
\hline 4.2.b. Asegurar la lógica del discurso \\
\hline 5. Manejo de la comprensión \\
\hline 5.1. Nivel de comprensión: ajuste a la consigna \\
\hline 5.3. Elaboración de conclusiones \\
\hline Estrategias identificadas \\
\hline
\end{tabular}


Interpretación de la información: El primer punto en la matriz de datos, corresponde a la Interpretación de la Información, entendida esta como la capacidad para seleccionar la información relevante, relacionarla con lo ya conocido y traducirla o codificarla a un nuevo código o lenguaje. Precisamente, analizando las respuestas brindadas por los alumnos en las situaciones problema y en las entrevistas, pudimos constatar que en general son capaces de seleccionar las ideas fundamentales. Esto lo efectúan de diversas formas: algunos sólo leyendo, otros subrayando, otros asociando lo planteado con lo ya conocido sobre el tema. En las entrevistas, algunos explicitan la forma cómo lograban extraer lo esencial de un texto, decodificándolo, traduciéndolo a su propio lenguaje, para luego realizar el proceso inverso y así responder a lo solicitado en las preguntas:

Entrevista Alumno 1:

"Primero leía la pregunta y me fijaba si se relacionaba con algo que yo había leído antes o se había dado en clase o con algo que sabía, algún juego o algo, lo que supiera relacionado a eso".

Entrevista Alumno 4:

"El de la 1 me fui guiando por lo que decía el texto para resolverlas. Pero los pasos, lo que decía ahí, trataba de hacerlo lo más concreto posible con lo que pedía la pregunta".

Comprensión y organización conceptual de la información: nos estamos refiriendo a la capacidad de entender, concebir, discernir, descifrar, justificar, con la finalidad de construir estructuras conceptuales concretas, coherentes. Así entendida, esta categoría implica no sólo destacar las palabras o ideas clave, sino también descubrir lo no conocido, formular hipótesis, inferir, relacionar y jerarquizar conceptos. Esto lo vemos ejemplificado en la respuesta que da el alumno 4 en el siguiente ejemplo:

\section{- ¿Cómo quedó tu pollito al final?}

- Lo que quise representar fue que... porque acá decía que el pollo iba creciendo a medida que pasaban los años respecto a la vaca. Porque lo que yo entendi fue que antes se consumía mucho más carne de vaca que pollo y ahora es al revés, se consume más pollo que carne. Acá puse que dentro de algunos años va a ser más grande el pollo que la vaca. Eso fue lo que quise poner.

Búsqueda de analogías para interpretar los fenómenos: En el punto 3 de nuestra matriz de análisis de datos aparece la búsqueda de analogías entendida, como la capacidad de explicar un fenómeno a través de un modelo semejante y válido, porque no siempre la analogía es fiel reflejo del fenómeno que se pretende representar. Esta es precisamente, una de las estrategias que esperábamos identificar y que sin embargo no se detectó.

Comunicación de la información: En el punto 4 nos referimos a la comunicación de la información concebida como la capacidad para expresarse mediante el uso de diversos códigos (lingüístico, iconográfico, gráfico) estrechamente relacionada al proceso de aprendizaje conceptual, ya que es difícil poder comunicar con claridad una idea si no existe precisión conceptual. Implica así mismo introducir el tema, respetar las reglas del código lingüístico, asegurar la lógica del discurso, producir argumentos pertinentes, extraer conclusiones, citar ejemplos. En la producción de los alumnos vemos que esta estrategia presenta limitaciones, tanto a nivel de la expresión oral como escrita. La puntuación y la sintaxis se ven alteradas como ilustramos con el siguiente ejemplo:

Alumno 5

"¿Qué dificultades encontraste al leer los ejercicios?

No, ninguna. Yo que sé. La primera en el ejercicio número 2, que yo no entendí mucho, así... y esta menos, la número 3. No sé, porque ese diez a la menos nueve... no entendí nada. Y después no encontré ninguna dificultad". 
El manejo de la comprensión: cuando planteamos este punto aludimos fundamentalmente a las estrategias metacognitivas, es decir al control y regulación de la comprensión. Este aspecto pudimos profundizarlo un poco más a través de las entrevistas, puesto que es en ellas donde los alumnos pudieron explicitar más y mejor, si habían entendido o no, qué dificultades concretas encontraron, ver si lo expuesto en las pruebas escritas coincide o no con las ideas que oralmente expone el estudiante entrevistado. Así mismo calibrar el nivel de conciencia que cada uno tiene de los inconvenientes encontrados en la resolución de los problemas. Así por ejemplo, mientras algunos educandos tienen plenamente asumidas sus carencias y/o dificultades, otros no se dan cuenta de que no entendieron. A modo de ejemplo transcribimos dos situaciones tomadas de un mismo alumno:

Entrevista Alumno 2

- ¿Qué dificultades encontraste al leer los ejercicios?

- Lo que más me costó fue la letra del ejercicio 3 que no lo pude hacer.

- ¿Qué, de la letra te generó más dificultad?

- Palabras precisas como nanogramo que no la conozco.

\section{Conclusiones}

El hecho de abordar las conclusiones no significa que estemos terminando este trabajo; muy por el contrario, sentimos que estamos retornando a una etapa inicial en la que diversas preguntas nos asediaban insistentemente. Desde un comienzo percibimos que nos adentrábamos en un tema tan apasionante como difícil de desentrañar. El aprendizaje, y por ende las estrategias de aprendizaje han sido, son y seguirán siendo aún por algún tiempo, un verdadero misterio. Misterio del que cada osado investigador podrá deshilar apenas algún filamento de la rica y apretada trama que constituyen el no menos complejo mundo de los procesos de enseñanza y de aprendizaje.

En distintas instancias, en nuestro marco teórico, hablamos de una permanente dialéctica entre los involucrados en los procesos de enseñanza y de aprendizaje, así como también de la evolución del concepto de ciencia, de la incidencia del contexto y del momento histórico. Todo esto, nos ubica ante un fenómeno claramente multidimensional, en el que las explicaciones no pueden centrarse en un único aspecto, por lo que en la interpretación de los datos intentamos considerarlos a todos. Y es precisamente a la luz de los resultados obtenidos en la aplicación de las situaciones problema y de las entrevistas - que al ser un estudio de caso no nos permite realizar generalizaciones- que podríamos decir que se detectan en estos alumnos de $3^{\text {er }}$ año del Ciclo Básico, más estrategias de las que a priori esperábamos. La mayoría de los estudiantes entrevistados demostraron ser capaces de leer y comprender con acierto lo planteado, pasar de un código a otro, argumentar en forma coherente, relacionar la nueva información con lo ya conocido, deducir, inferir, predecir. Sin embargo, si tuviésemos que calificar los trabajos, tal como lo hacemos habitualmente en las pruebas escritas, los resultados no serían aceptables, a excepción tal vez de un alumno. Esta realidad nos remonta al propósito de nuestra investigación, cuando transcribimos la opinión de muchos de nuestros colegas: "los alumnos no saben estudiar", "los estudiantes no comprenden lo que leen", "solos no consiguen razonar y resolver los problemas que se les presentan". Esta percepción de los docentes, puede tener distintas explicaciones. Así como los alumnos tienen sus representaciones respecto de los docentes y de los temas a abordar, también los profesores tienen sus preconcepciones acerca de los estudiantes, de cómo aprenden; igualmente basadas en experiencias de la vida cotidiana práctica educativa- en el refuerzo de la cultura y del lenguaje habitual. 
Pensamos que las opiniones vertidas sobre los educandos responden más que a un análisis de las estrategias de aprendizaje a una medición de su desempeño, de su rendimiento; se toma en cuenta el producto y no el proceso. No se les pide que expliciten cómo llegaron a cierta respuesta y se evalúa en consecuencia. Por otra parte, la confusión entre estrategia y técnica puede cooperar en la concepción del docente, ya que comúnmente se utilizan como sinónimos. Sin embargo, de acuerdo con nuestro marco conceptual, la estrategia requiere la toma de conciencia y regulación en el empleo de la misma, de parte de quien la utiliza. Lo expresado, nos pone justo en la antesala de otra incógnita: ¿Por qué los alumnos, en la resolución de problemas no utilizan siempre que sean necesarias, las estrategias que aparentemente habrían logrado? Tal vez parte de la explicación esté dada por lo que afirma Meirieu: "La estrategia de un sujeto se articula de este modo en un estilo cognitivo personal relativamente estable pero que depende también del objeto de aprendizaje" (1992:204). Esto justifica que el logro de estrategias tenga diferentes niveles de adquisición, relacionados con la edad de los alumnos, ya que la adolescencia es una etapa de inestabilidad en los procesos, de ahí que podamos entender la diversidad y la aparente discrepancia en la posesión de las estrategias. También la falta de interés, de deseo y de compromiso con la tarea impedirían el logro de la mencionada estabilidad. Además, a la diversidad individual, deberíamos sumar el hecho de que en la concepción teórica planteada, el alumno pasa a ser el protagonista cumpliendo un rol activo, lo que potenciaría aún más la variabilidad en los procesos cognitivos y metacognitivos ya que se conjugan: la historia personal, las vivencias, los valores y las creencias de cada persona. Pero además, aunque nuestro estudio no se efectúa desde la Didáctica, no podemos dejar de pensar en la relevancia del rol del docente que es en definitiva el que mostrará a los alumnos distintas técnicas y estrategias de enseñanza, generará espacios de reflexión, de autoevaluación, propondrá situaciones problema para que los estudiantes descubran y pongan en práctica las estrategias cognitivas y metacognitivas. Es por este motivo, que en nuestra propuesta inicial, habíamos planteado la necesidad de efectuar la observación de clase, que por falta de tiempo no pudimos realizar.

En suma, el análisis de las estrategias de aprendizaje en adolescentes de 3er. año del Ciclo Básico constituye un fenómeno multidimensional, en el que todos y cada uno de los aspectos involucrados deben ser tenidos en cuenta. No encontramos una respuesta contundente, definitiva, sino que se produce un giro epistémico que nos lleva en cambio a formularnos nuevas preguntas:

- ¿Será que la falta de conocimientos específicos en un área de conocimiento, bloquea las estrategias?

-La formación recibida por los alumnos, ¿es la adecuada para la regulación efectiva de su propio proceso de aprendizaje y el logro de las estrategias?

- ¿Será que ellos poseen estrategias desconocidas para nosotros, que provienen de un aprendizaje y/o razonamiento informal?

Dado que aún no podemos responder estas interrogantes, invitamos a otros investigadores a continuar indagando con nosotras, para llegar a desentrañar los "misterios" que envuelven este tema tan atrapante como complejo. Asimismo, desearíamos que nuestros colegas -los docentes- tuviesen la posibilidad de reflexionar sobre su práctica educativa y su incidencia en el proceso de aprendizaje de los estudiantes, tal como la tuvimos nosotras, en ocasión de elaborar este trabajo, que nos ha resultado altamente enriquecedor por lo que aprendimos, y altamente gratificante por lo que significó el intercambio con otros docentes con inquietudes similares a las nuestras y que a su vez nos permitieron conocer perspectivas diferentes sobre una amplia variedad de temas. 


\section{Bibliografía}

AVOLIO de COLS, S. 1998. Los proyectos para el trabajo en el aula. Buenos Aires: Marymar.

CARRETERO, M. 1993. Constructivismo y educación. Buenos Aires: Aique.

CHALMERS, A.F. 1998. ¿Qué es esa cosa llamada ciencia? Buenos Aires: Siglo XXI.

CONTRERAS, J. 1994. Enseñanza, currículum y profesorado. Madrid: Akal.

FUMAGALLI, L. 1998. EI desafío de enseñar ciencias naturales. Buenos Aires: Troquel.

GIBAJA, R. 1995. El trabajo intelectual en la escuela. Buenos Aires: Academia Nacional de Educación. GIROUX, H. y Mc. LAREN, P. 1998. Sociedad, cultura y educación. Madrid: Miño y Dávila.

HUME, D. 1984. Investigación sobre el conocimiento humano. Madrid: Alianza.

JONES, F, y otros. 1987. Estrategias para enseñar a aprender. Buenos Aires: Aique.

MAYOR, J. y otros. 1995. Estrategias Metacognitivas. Madrid: Editorial Síntesis SA.

MEIRIEU, P. 1992. Aprender, sí. Pero ¿cómo? Barcelona: Ediciones Octaedro.

MINNICK SANTA, C. y ALVERMAN, D. 1994. Una didáctica de las ciencias. Buenos Aires: Aique. MONEREO, C. y otros. 1998. Estrategias de Enseñanza y Aprendizaje. 5ta. Edición. Barcelona: Grao.

OBIOL, G. y Di SEGNI de OBIOLS, S. s/d. Adolescencia, posmodernidad y escuela secundaria. Buenos Aires: Kapeluz Editora SA.

POZO, J. I. y MONEREO, C. 1999. El aprendizaje estratégico. Madrid: Santillana.

POZO, J. I.. 1996. Aprendices y Maestros. La nueva cultura del aprendizaje. Madrid: Alianza Editorial.

POZO, J. I.. 1998. Aprender y enseñar ciencias. Madrid: Morata.

TEDESCO, J. 1999. El nuevo impacto educativo. Madrid: Grupo ANAYA SA.

TAYLOR, S. y BOGDAN, R. 1987. Introducción a los métodos cualitativos en investigación.

Buenos Aires: Paidós

VIGOTSKY, L. 1992. Pensamiento y Lenguaje. Buenos Aires: La Pléyade.

*,** y *** Diploma en Educación, Universidad ORT Uruguay. 